\title{
THE CORE PROPERTIES OF ELLIPTICAL GALAXIES
}

\author{
Tod R. Lauer, \\ Princeton University Observatory \\ Princeton, NJ 08540, USA
}

\begin{abstract}
The core structure of elliptical galaxies is determined by two parameters: total luminosity $L$ and central luminosity density $\rho_{C}$. Scatter in $\rho_{C}$ at any $L$ may imply dissipational formation of core structure; once this scatter is accounted for, however, the pure $L$ dependence of the core parameters can be isolated and perhaps used as a metric distance indicator.
\end{abstract}

\section{DISCUSSION}

Lauer (1985) and Kormendy (1985) find similar core parameter relationships as a function of luminosity. Brighter galaxies tend to have bigger and dimmer cores specifically I get $r_{C} \propto L^{1.2}$ and $I_{0} \propto L^{-1.0}$, where $r_{C}$ and $I_{0}$, are the core radius and central surface brightness. There is large cosmic scatter in both relationships, however, but with tightly correlated residuals; at any luminosity excessively large cores are dim in the center and excessively compact cores are brighter; this can be attributed to even larger scatter in core luminosity densities. Over an order of magnitude variation is observed in $\rho_{C}$ at any luminosity; this implies that $L$ alone is not the sole parameter determining a galaxy's core structure.

I believe the scatter in $\rho_{C}$ suggests dissipational formation of the cores. Cores of giant ellipticals contain only $10^{8}-10^{9} M_{\odot}$, a small fraction of their total masses. Further, since the cores sit at the bottom of their galaxies' potential wells they are sensitive to the central accretion of even small amounts of matter which would otherwise have no effect on the global structure of their galaxies - it is interesting to note that there appears to be no relationship between the ratio $R_{E} / r_{C}$ and galaxy luminosity. This argues against the formation of elliptical galaxies by any sort of dissipationless hierarchy of merging, since simulations of such processes predict $R_{E} / r_{C}$ to increase as a strong function of $L$. Merging with dissipation might be expected to produce even more compact cores for a given final effective radius; however, variations in the amount of "stuff" allowed to sink to the center of the final merger product might obscure any basic dependence of $R_{E} / r_{C}$ on $L$.

Once the scatter in $r_{C}$ and $I_{0}$ at any luminosity is understood as being caused by variations in $\rho_{C}$, it is possible to isolate the pure luminosity dependence of the core parameters. A principal components analysis gives $L \propto r_{C}^{2.4} I_{0}^{1.7}$. As argued by Dressler et al. (1986) central velocity dispersion $\sigma$ correlates better than $L$ with global structural properties of the gaiaxies; this also appears to be true with the core 


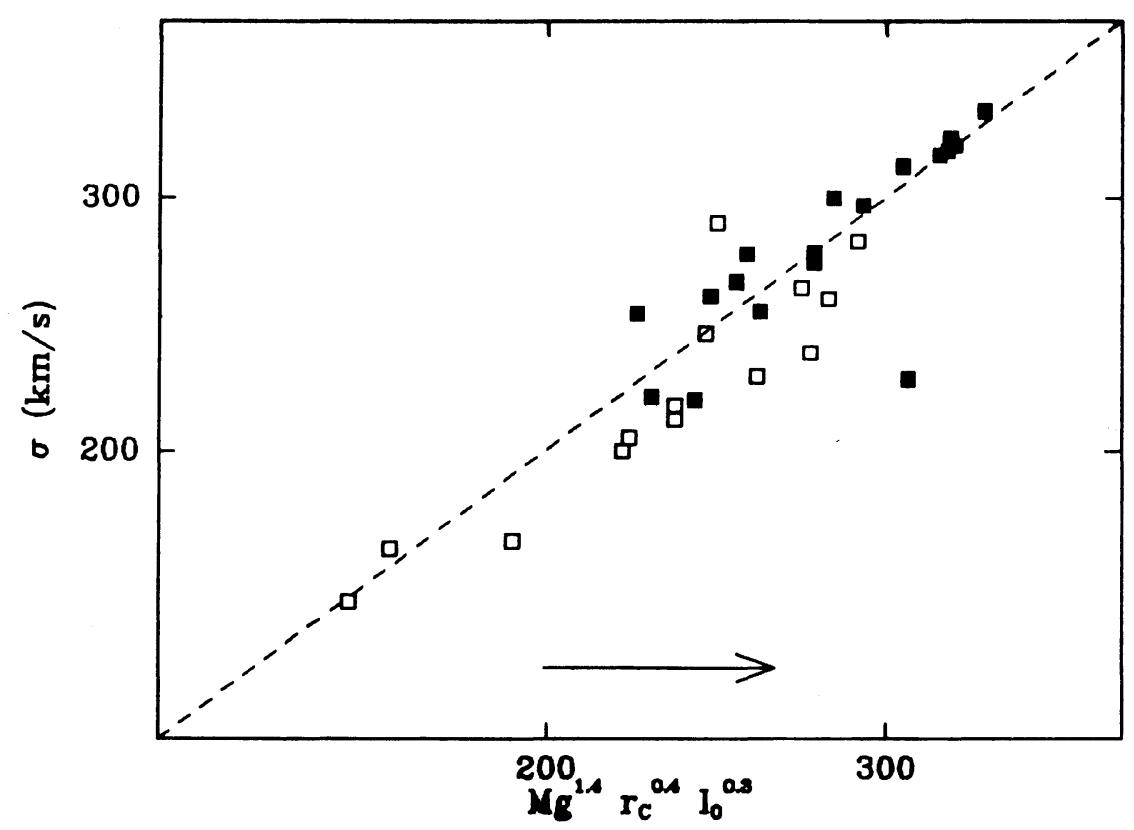

Figure 1: Central velocity dispersion plotted against that calculated from a fit to $r_{C}$, $I_{0}$ and $M_{2}$. Solid symbols are for galaxies with resolved cores. The arrow shows the change in calculated $\sigma$ for a factor-of-two change in $r_{C}$.

parameters and works best with the central $M g_{2}$ index tossed in. Figure 1 shows the results of a fit involving central velocity dispersion, $\sigma, r_{C}, I_{0}$, and $M g_{2}$ on the assumption that the later three variables determine $\sigma$. The resulting fit using just the well resolved galaxies gives:

$$
\sigma \propto r_{C}^{0.4} I_{0}^{0.3} 10^{1.4 M g_{2}} .
$$

Dispersions predicted from the core parameters of the resolved galaxies fall very close to their true values. Note that $r_{C}$ is the only distance dependent parameter in (1); the goodness of the fit suggests that cores might be used as a metric distance indicator if (1) can be confirmed with a larger sample of galaxies with resolved cores.

\section{REFERENCES}

Dressler, A., Lynden-Bell, D., Burstein, D., Davies, R. L., Faber, S. M., Terlevich, R. J., Wegner, G. 1986, preprint.

Kormendy, J. 1985, Ap. J., $295,73$.

Lauer, T. R. 1985, Ap. J., 292, 104. 